\title{
Prophylactic abdominal aortic balloon occlusion: An effective method of controlling hemorrhage in patients with placenta previa or accreta
}

\author{
ZHAO HONG PENG, ZHUANG XIONG, BEN SHENG ZHAO, GUO BING ZHANG, \\ WEN SONG, LONG XIANG TAO and XIU ZE ZHANG \\ Department of Interventional Radiology, The First Affiliated Hospital of \\ Anhui Medical University, Hefei, Anhui 230022, P.R. China \\ Received May 18, 2018; Accepted November 7, 2018
}

DOI: $10.3892 /$ etm.2018.7066

\begin{abstract}
Postpartum hemorrhage is considered to be a serious complication in patients with pernicious placenta. Approaches employing abdominal aortic balloon occlusion to control hemorrhage are extremely effective for such patients. The present study analyzed 9 patients with pernicious placenta previa in a single hospital from June 2016 to November 2017. Prior to cesarean hysterectomy, an abdominal aortic balloon catheter was placed in all patients. The balloon was inflated and evacuated alternately using saline following delivery of the fetal head. The X-ray dose, bleeding volume and complications during the procedure were observed. Balloon catheterization was successfully performed in all 9 patients. The dose of X-rays ranged from 15.8 to $24.5 \mathrm{mGy}$, with a mean of $19.3 \pm 2.7 \mathrm{mGy}$; the volume of blood loss ranged from 50 to $4,000 \mathrm{ml}$, with a mean of $\sim 1,800 \mathrm{ml}$. Uterine artery embolization was successfully performed in 2 cases due to bleeding following the cesarean hysterectomy, and every uterus was retained. Abdominal aortic balloon occlusion can effectively reduce the amount of bleeding during cesarean hysterectomy in patients with pernicious placenta previa. This may serve as technical support for patients in whom retention of the uterus is expected. However, it is necessary to identify any abnormal uterine tissue above the level of the renal artery in order to avoid ineffective balloon occlusion.
\end{abstract}

\section{Introduction}

Pernicious placenta previa occurs in patients who have previously delivered via cesarean section, and in whom

Correspondence to: Dr Zhuang Xiong, Department of Interventional Radiology, The First Affiliated Hospital of Anhui Medical University, 218 Jixi Road, Hefei, Anhui 230022, P.R. China E-mail: pzh52492@163.com

Key words: abdominal aorta, balloon occlusion, pernicious placenta previa, cesarean hysterectomy the placenta previa is attached to the scars of the previous uterine incision (1). The main clinical symptoms of placenta previa include irregular vaginal bleeding, hysterorrhexis, postpartum hemorrhage and invasion of the placenta into pelvic organs. Fatal postpartum hemorrhage is often a danger to maternal life (2). In this emergency situation, obstetricians may have to perform obstetric hysterectomy in order to save the patient's life.

Placenta accreta is the direct invasion of placental villi into myometrium (3). At present, the clinical treatment options for placenta previa and accreta, include internal iliac artery ligation, internal iliac artery embolization, uterine artery embolization, intrauterine balloon tamponade and wound suturing (4-6). The pelvic region is rich in collateral circulation and arterial anastomoses. Frequently, limited treatment outcomes are achieved from internal iliac artery ligation, intrauterine balloon tamponade and wound suturing (7). The methods of internal iliac artery embolization and uterine artery embolization were thought to be universally effective (8). However, only a minority of hospitals in China have hybrid operating rooms and, due to the life-threatening nature of postpartum hemorrhage or hemorrhagic shock, there may not be sufficient time for patients to be transferred from the surgical operating room to another operating room for digital subtraction angiography (DSA). Therefore, with regard to delayed postpartum hemorrhage or minor hemorrhage, we consider uterine artery embolization to be a better treatment choice.

Currently, there are few reports concerning the use of internal iliac artery balloon occlusion or abdominal artery balloon occlusion to control postpartum hemorrhage in patients with pernicious placenta previa $(6,9)$. The current study reports some successful experience and problems associated with the use of abdominal artery balloon occlusion in patients with pernicious placenta previa.

\section{Patients and methods}

Patients. The present retrospective study was conducted among women with pernicious placenta previa accreta/increta who were treated in The First Affiliated Hospital of Anhui Medical 


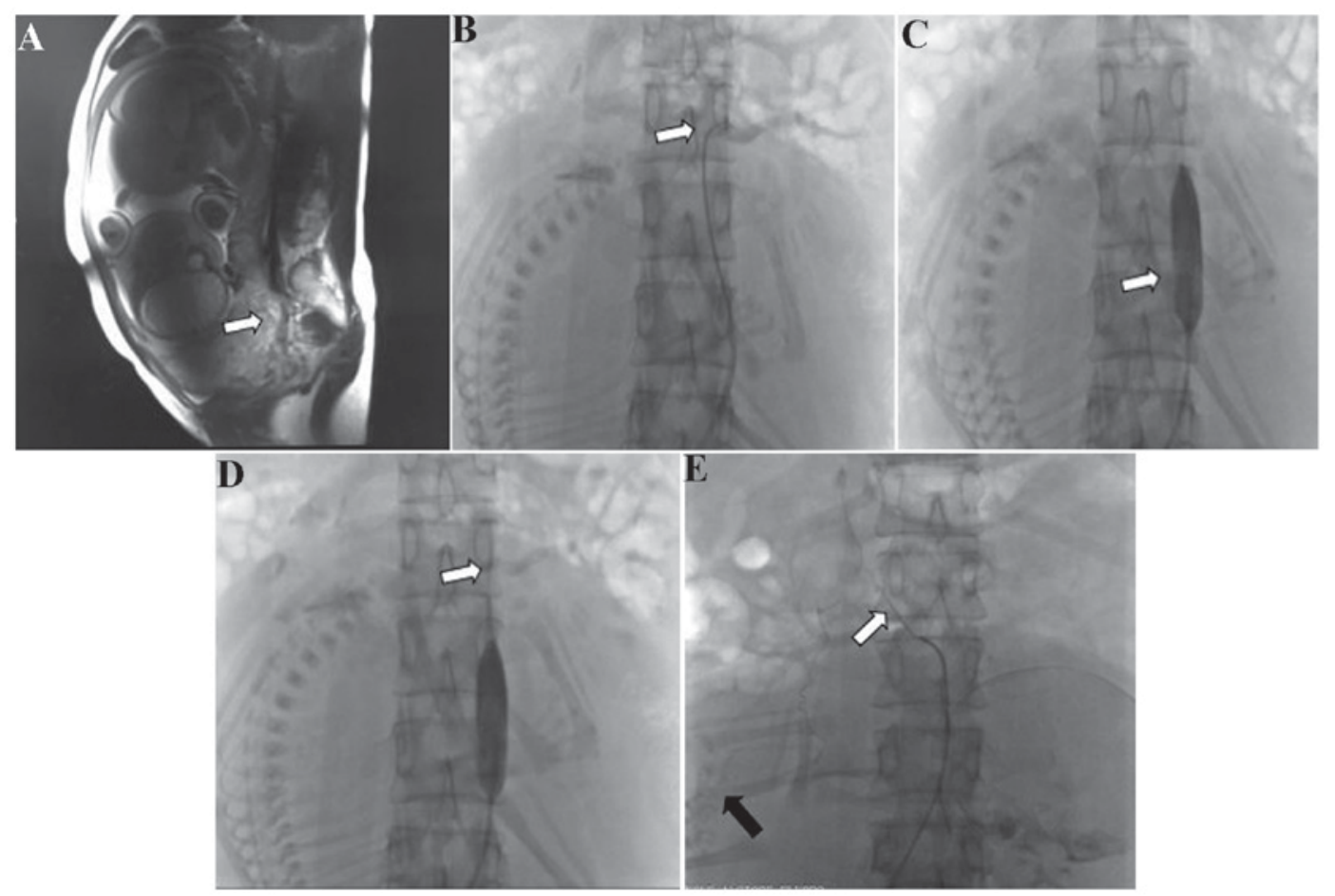

Figure 1. (A) Sagittal magnetic resonance image showing placental implantation in the myometrium of the uterus (white arrow). (B) Cobra catheterization with angiography to confirm the level of the renal artery (white arrow). (C) Angiography demonstrating insertion of the balloon catheter into the abdominal aorta below the level of the renal artery (white arrow). (D) Balloon catheterization with angiography to confirm that blood flow was blocked. (E) Cobra catheterization with angiography, identifying that the uterine artery (black arrow) originated from the adrenal artery (white arrow).

University (Hefei, China) from June 2016 to November 2017. A total of 9 patients were diagnosed with placenta previa accreta/increta by color Doppler ultrasonography and/or magnetic resonance imaging (10). The age range of the patients was 23-37 years (mean age, 29.8 years). All patients gave written informed consent for the use of their data in clinical research, and the study was approved by the Ethics Committee of Anhui Medical University.

Prior to cesarean delivery, all patients and their families were informed of the risks of the procedure, the details of the abdominal aortic balloon occlusion and cesarean section, and the possible complications of abdominal aortic balloon occlusion during cesarean delivery. It was necessary to perform magnetic resonance imaging (MRI; Fig. 1A) or ultrasonography to ascertain the scope and position of the placenta previa accreta/increta. At the same time, measurements of the diameter of the abdominal aorta were made according to the results of the MRI or ultrasonography, in order to select the appropriate balloon size.

Interventional procedure. On the day of cesarean delivery, in the DSA operating room, puncture of the right femoral artery and placement of an $8 \mathrm{~F}$ arterial sheath (Bard Medical Division, Covington, GA, USA) was performed in each patient following local anesthesia using 2\% lidocaine. Under fluoroscopic guidance, a 5F cobra or pigtail catheter (Terumo Co., Ltd., Tokyo, Japan) was placed into the abdominal aorta under angiography using a guide wire to clear the renal artery; the cobra or pigtail catheter was removed when the opening level of the renal artery was identified. Similarly, a balloon catheter was placed into the abdominal artery using a guide wire, with the balloon positioned below the level of the renal artery (Fig. 1B-D). Following X-ray fluoroscopy, the X-ray dose was recorded.

Cesarean procedure. Following the interventional procedure, the patient was transferred to the surgical operating room to undergo the cesarean procedure. Once the fetal head had been delivered, the balloon was inflated with $0.9 \%$ saline $(15 \mathrm{ml})$ to block blood flow immediately. The balloon was inflated for no longer than $15 \mathrm{~min}$, and was then was deflated for $3 \mathrm{~min}$. Inflation and deflation were alternated until no evident bleeding was observed and the uterine wound was then sutured completely. The balloon catheter was withdrawn following the completion of the whole procedure. The arterial sheath was removed after $6 \mathrm{~h}$. The estimated blood loss, the volume of blood products transfused, the duration of the surgical procedure and the Apgar score at 5 min were recorded (11). Patients were monitored for complications for $24 \mathrm{~h}$ after surgery.

\section{Results}

All observation data are summarized in Table I. The procedure was completely successful in 7 patients, in whom the maximum estimated volume of blood loss was $\leq 2,500 \mathrm{ml}$. Embolic uterine arterial occlusion was required in the other 2 cases due to uncontrolled postpartum hemorrhage. In one of these cases, the estimated blood loss was 4,000 ml. Notably, during the abdominal aortic angiography procedure, it was observed that the uterine artery originated from the adrenal artery in this case (Fig. 1E). In the other case, the estimated blood loss was 3,500 $\mathrm{ml}$. During the cesarean delivery procedure, this patient was observed to have a high degree of abnormal 


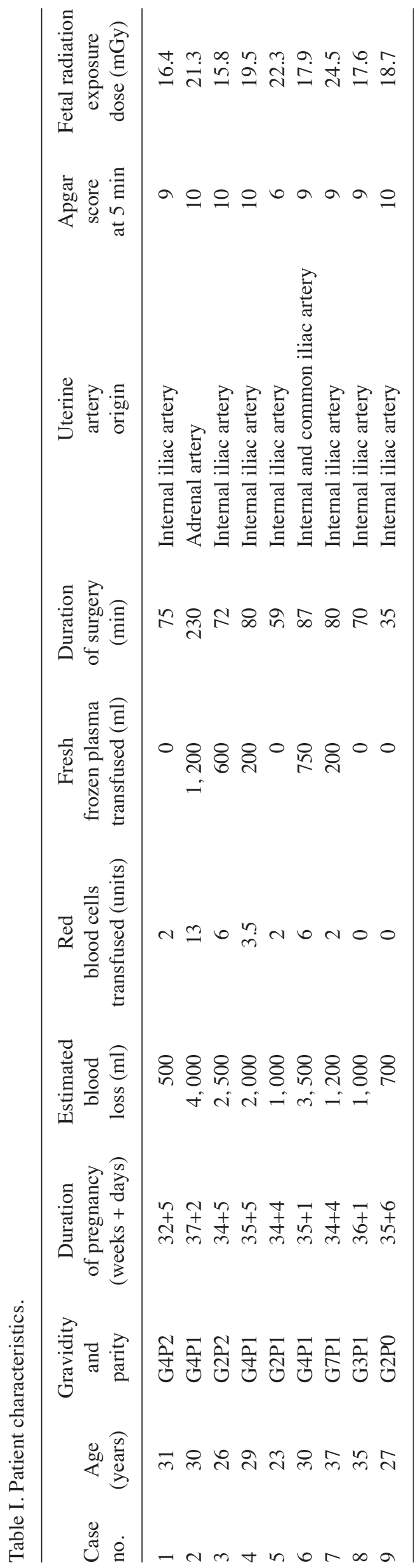

placental implantation. During the uterine arterial angiography and embolization, the presence of double uterine arteries was identified, with one originating from the internal iliac artery and another from the common iliac artery. In the study group, the total radiation dose to which the fetus was exposed was 15.8-24.5 mGy, with a mean of $19.3 \pm 2.7 \mathrm{mGy}$ (Table I). The largest transfusion was received by was patient 2 and the mean number of units of red blood cells transfused was 3.83. Similarly, fresh frozen plasma transfuse was positively associated with the transfusion of red blood cells. The range of duration of surgery was 35-230 min and the mean was 87.6 min. The Apgar score at 5 min range was 6-10 and the mean was 9.1. All patients retained the uterus successfully in the current study, without the occurrence of severe complications.

\section{Discussion}

Accurate diagnosis of pernicious placenta previa is very important in the formulation of a detailed plan for subsequent cesarean delivery. At present, ultrasonography and MRI serve an important role in the identification of placenta previa accreta/increta (12). Three-dimensional color Doppler ultrasound scans have a high sensitivity and specificity in the diagnosis of pernicious placenta previa, as they can identify abnormal bleeding from the placental bed and discern the chorionic villi from the myometrium (10). MRI of the soft tissue has marked advantages, as it may allow clinicians to detect whether chorionic villi have invaded into the myometrium and/or peripheral organs/tissues (10). In the present study, 2 patients were definitively diagnosed by ultrasonography, and 7 patients by MRI. During the examination, the diameter of the abdominal aorta was also measured in order to determine the optimum balloon size. The selection of an appropriate balloon is essential; if the balloon is oversized, it may cause injury to the abdominal arterial endangium during the process of inflation; if it is too small, it will not be able to effectively control the bleeding during the cesarean procedure.

Cesarean delivery has been the treatment strategy for placenta previa for many years (13). However, the control of intractable bleeding during the surgery is key to the treatment. Traditional methods of reducing blood loss include changing the uterine suture method, intrauterine tamponade using a balloon or gauze, uterine artery or internal iliac artery ligation, and uterine artery or internal iliac artery embolization (5,14-16). A number of previous studies have reported that prophylactic abdominal aortic or internal iliac artery balloon catheter application reduces bleeding successfully during cesarean delivery in patients with placenta previa $(6,17-19)$. However, internal iliac artery balloon occlusion requires the use of two balloons and bilateral femoral artery puncture; this increases the economic burden for the patient and is inconvenient owing to the puncture points requiring pressure homeostasis following the procedure. Therefore, in the present study, abdominal aortic balloon occlusion was used as the method of reducing blood loss during the cesarean delivery, and was demonstrated to be successful in the majority of the patients with placenta previa. The minimum estimated blood loss was $500 \mathrm{ml}$, which is similar to that reported in studies by Xie et al (18) and Sun et al (19). Although it may be useful in the majority of patients, the authors of the current 
study hypothesised that the abdominal aortic balloon occlusion method might not be completely effective in cases with an ectopic uterine artery, large-area placental implantation or poor uterine contraction. In the present study, the uterine artery of one patient was derived from the adrenal artery. The estimated blood loss was $4,000 \mathrm{ml}$ during the cesarean delivery in this case, demonstrating the method to be almost ineffective; the balloon position was located below the opening of the renal artery in order to avoid renal injury. The estimated blood loss for another patient was 3,500 ml due to large-area placental implantation. Fortunately, uterine artery embolization was subsequently successfully performed in these cases. A previous study by Sun et al (19) reported a patient whose estimated blood loss was $9,000 \mathrm{ml}$. We suspect this patient may have had an anomalous uterine artery.

Complications associated with arterial balloon application have been reported in previous studies $(6,18-22)$, including ischemic necrosis of the lower limbs, internal iliac arterial thrombosis, puncture point hematoma, reperfusion injury of tissues and organs, acute renal failure and initial vessel injury. In the studies conducted by Xie et al (18) and Sun et al (19), a $12 \mathrm{~F}$ arterial sheath was used. It is evident that larger arterial sheaths create a larger femoral arterial lesion at the puncture site. When pulling out an arterial sheath, a larger lesion will make compression and hemostasis more difficult, and a hematoma may easily form at the puncture site. In the study of Xie et al (18), a patient presented a hematoma in the right common femoral artery following arterial sheath removal. In the present study, the appropriate arterial sheath was chosen according to the size of the balloon catheter. The maximum arterial sheath was $<10 \mathrm{~F}$. No complications occurred in any of the patients. We suggest that the choice of balloon size and control of the balloon occlusion time are important factors. If the balloon is too large, it will cause abdominal aortic injury, and if the balloon occlusion time is too long, it will cause thrombosis of the lower limb and reperfusion injury. The optimal occlusion time has been suggested to be in the range of $25-80 \min (18,19,23,24)$. However, in the study by Sun et al (19), one patient developed thrombosis in the internal iliac artery following surgery in which a $20-\mathrm{mm}$ balloon was selected and $\leq 40$-min periods of occlusion with intervals of $10 \mathrm{~min}$ were used. In the present study, the diameter of the abdominal aorta was measured by MRI or ultrasonography prior to surgery to determine the appropriate size of the balloon catheter. Generally, the diameter of the selected balloon was $\leq 2 \mathrm{~mm}$ wider than the diameter of the abdominal aorta. Furthermore, occlusion was maintained for no longer than $15 \mathrm{~min}$ at a time with intervals of $3 \mathrm{~min}$, and no complications occurred in any of the patients. Therefore, measurement of the abdominal aortic diameter prior to intervention, clearance of the opening of the renal artery and control of the duration of balloon occlusion are indicated to be important for decreasing the risk of complications.

Additionally, minimizing the fetal radiation exposure dose is an important factor when considering these methods. The latest report about mean fetal radiation exposure dose recommended a dose of $4.2 \pm 1.9 \mathrm{mGy}$ (19). However, we consider it difficult to achieve this target, particularly when using DSA. The X-ray dose of DSA far exceeds that of fluoroscopy at the same exposure time (25). In the present study, the mean fetal radiation exposure dose was $19.3 \pm 2.7 \mathrm{mGy}$, which is far less than the standard dose of $\leq 150 \mathrm{mGy}$ recommended by the National Committee on Radiological Protection (26). Insertion of the balloon into the aorta was performed rapidly in all patients by experienced interventional radiologists to minimize radiation exposure.

In conclusion, the prophylactic use of abdominal aortic balloon catheters is a relatively safe and effective method of treating patients with placenta previa, which may control hemorrhaging during cesarean delivery and reduce the risk of hysterectomy. However, the control of balloon occlusion-associated complications requires further consideration. The present study had certain limitations, such as a small sample size and the absence of a control group. A control group was not included in the current study because the method used was demonstrated to be very effective in previous studies $(18,19)$. However, since uncontrolled postpartum hemorrhage occurred in cases with abnormal uterine arteries in the present study, it was considered necessary to report these findings in a timely manner in order to avoid ineffective balloon occlusion.

\section{Acknowledgements}

Not applicable.

\section{Funding}

No funding was received.

\section{Availability of data and materials}

All data generated or analyzed during this study are included in this published article.

\section{Authors' contributions}

ZHP, ZX, BSZ, GBZ, WS, LXT and XZZ performed the surgery, analyzed the data and prepared the manuscript. ZHP and $\mathrm{ZX}$ were responsible for study conception and design.

\section{Ethics approval and consent to participate}

All patients gave written informed consent for the use of their data in clinical research. The current study was approved by the Ethics Committee of Anhui Medical University.

\section{Patient consent for publication}

Written informed consent was provided for publication of the patients' data.

\section{Competing interests}

The authors declare that they have no competing interests.

\section{References}

1. He Y and Chen D: New understanding of the diagnosis and management of pernicious placenta previa. Chin J Perinat Med 18: 494-496, 2015 (In Chinese). 
2. Sumigama S, Itakura A, Ota T, Okada M, Kotani T, Hayakawa H, Yoshida K, Ishikawa K, Hayashi K, Kurauchi O, et al: Placenta previa increta/percreta in Japan: A retrospective study of ultrasound findings, management and clinical course. J Obstet Gynecol Res 33: 606-611, 2007.

3. Silver RM and Barbour KD: Placenta accreta spectrum: Accreta, Increta, and percreta. Obstet Gynecol Clin North Am 42: 381-402, 2015.

4. Chen ZY, Li J, Shen J, Jin J, Zhang W and Zhong W: Direct puncture embolization of the internal iliac artery during cesarean delivery for pernicious placenta previa coexisting with placenta accreta. Int J Gynecol Obstet 135: 264-267, 2016.

5. Xu JQ: Effectiveness of embolization of the internal iliac or uterine arteries in the treatment of massive obstetrical and gynecological hemorrhages. Eur Rev Med Pharmacol Sci 19: 372-374, 2015.

6. Cui SH, Zhi YX, Cheng GM, Zhang K, Zhang L and Shen L: Retrospective analysis of placenta previa with abnormal placentation with and without prophylactic use of abdominal aorta balloon occlusion. Int J Gynecol Obstet 137: 265-270, 2017.

7. Arduini M, Epicoco G, Clerici G, Bottaccioli E, Arena S and Affronti G: B-Lynch suture, intrauterine balloon, and endouterine hemostatic suture for the management of postpartum hemorrhage due to placenta praevia accrete. Int J Gynaecol Obstet 108: 191-193, 2010.

8. Ganguli S, Stecker MS, Pyne D, Baum RA and Fan CM: Uterine artery embolization in the treatment of postpartum uterine hemorrhage. J Vasc Interv Radiol 22: 169-176, 2011.

9. Carnevale FC, Kondo MM, de Oliveira Sousa W Jr, Santos AB, da Motta Leal Filho JM, Moreira AM, Baroni RH, Francisco RP and Zugaib M: Perioperative temporary occlusion of the internal iliac arteries as prophylaxis in cesarean section at risk of hemorrhage in placenta accreta. Cardiovasc Intervent Radiol 34 758-764, 2011.

10. Ayati S, Leila L, Pezeshkirad M, Seilanian Toosi F, Nekooei S, Shakeri MT and Golmohammadi MS: Accuracy of color Doppler ultrasonography and magnetic resonance imaging in diagnosis of placenta accreta: A survey of 82 cases. Int J Reprod Biomed (Yazd) 15: 225-230, 2017.

11. Crawford JS: The Apgar scoring system. Dev Med Child Neurol 4: 441-444, 1962.

12. Balcacer P,Pahade J, Spektor M, Staib L, Copel JA and McCarthy S: Magnetic resonance imaging and sonography in the diagnosis of placental invasion. J Ultrasound Med 35: 1445-1456, 2016.

13. Deng L, Chang Q, Wang Y, Wang L, Li Y and Hu Q: Tourniquet device for hemorrhage control during cesarean section of complete placenta previa pregnancies. J Obstet Gynaecol Res 40: 399-404, 2014

14. Danisman N, Kahyaoglu S, Celen S, Akselim B, Tuncer EG, Timur H, Kaymak O and Kahyaoglu I: The outcomes of surgical treatment modalities to decrease 'near miss' maternal morbidity caused by peripartum hemorrhage. Eur Rev Med Pharmacol Sci 18: 1092-1097, 2014.
15. Kaya B, Tuten A, Daglar K, Onkum M, Sucu S, Dogan A, Unal $\mathrm{O}$ and Guralp O: B-Lynch uterine compression sutures in the conservative surgical management of uterine atony. Arch Gynecol Obstet 291: 1005-1014, 2015

16. Ko HK, Shin JH, Ko GY, Gwon DI, Kim JH, Han K and Lee SW: Efficacy of prophylactic uterine artery embolization before obstetrical procedures with high risk for massive bleeding. Korean J Radiol 18: 355-360, 2017.

17. Angileri SA, Mailli L, Raspanti C, Ierardi AM, Carrafiello G and Belli AM: Prophylactic occlusion balloon placement in internal iliac arteries for the prevention of postpartum haemorrhage due to morbidly adherent placenta: Short term outcomes. Radiol Med 122: 798-806, 2017.

18. Xie L, Wang Y, Luo FY, Man YC and Zhao XL: Prophylactic use of an infrarenal abdominal aorta balloon catheter in pregnancies complicated by placenta accreta. J Obstet Gynaecol 37: 557-561, 2017.

19. Sun WJ, Duan SH, Xin G, Xiao J, Hong F, Hong H, Wu Y and $\mathrm{Xu}$ Y: Safety and efficacy of preoperative abdominal aortic balloon occlusion in placenta increta and/or percreta. J Surg Res 222: 75-84, 2018.

20. Bishop S, ButlerK, Monaghan S, Chan K, Murphy G andEdozien L: Multiple complications following the use of prophylactic internal iliac artery balloon catheterisation in a patient with placenta. Int J Obstet Anesth 20: 70-73, 2011.

21. Sewell MF, Rosenblum D and Ehrenberg H: Arterial embolus during commom iliac balloon catheterization at cesarean hysterectomy. Obstet Gynecol 108: 746-748, 2006.

22. Wei X, Zhang J, Chu Q, Du Y, Xing N, Xu X, Zhou Y and Zhang W: Prophylactic abdominal aorta balloon occlusion during caesarean section: A retrospective case series. Int J Obstet Anesth 27: 3-8, 2016.

23. Masamoto H, Uehara H, Gibo M, Okubo E, Sakumoto K and Aoki Y: Elective use of aortic balloon occlusion in caesarean hysterectomy for placenta previa percreta. Gynecol Obstet Invest 67: 92-95, 2009

24. Andoh S, Mitani S, Nonaka A, Suzuki S, Tamaki F, Ohmori K and Kimura E: Use of temporary aortic balloon occlusion of the abdominal aorta was useful during cesarean hysterectomy for placenta accrete. Masui 60: 217-219, 2011 (In Japanese)

25. Harrington DP, Boxt LM and Murray PD: Digital subtraction angiography: Overview of technical principles. AJR Am J Roentgenol 139: 781-786, 1982.

26. The 2007 recommendations of the international commission on radiological protection. ICRP publication 103. Ann ICRP 37: 1-332, 2007. 